\title{
Bounded Rationality of Generalized Abstract Fuzzy Economies
}

\author{
Lei Wang ${ }^{1}$ and Yu Fu ${ }^{2,3}$ \\ ${ }^{1}$ School of Economic Mathematics, Southwestern University of Finance and Economics, Chengdu, Sichuan 610074, China \\ ${ }^{2}$ School of Business Administration, Southwestern University of Finance and Economics, Chengdu, Sichuan 610074, China \\ ${ }^{3}$ School of Economic and Administration, Sichuan University of Science and Engineering, Zigong, Sichuan 643000, China \\ Correspondence should be addressed to Lei Wang; leiwang@swufe.edu.cn
}

Received 24 February 2014; Accepted 24 March 2014; Published 14 April 2014

Academic Editor: Nan-Jing Huang

Copyright (C) $2014 \mathrm{~L}$. Wang and Y. Fu. This is an open access article distributed under the Creative Commons Attribution License, which permits unrestricted use, distribution, and reproduction in any medium, provided the original work is properly cited.

By using a nonlinear scalarization technique, the bounded rationality model $M$ for generalized abstract fuzzy economies in finite continuous spaces is established. Furthermore, by using the model $M$, some new theorems for structural stability and robustness to $(\lambda, \epsilon)$-equilibria of generalized abstract fuzzy economies are proved.

\section{Introduction}

In 1952, Arrow and Debreu [1] first proved the existence theorem of the Walrasian equilibrium. Borglin and Keiding [2] generalized Arrow and Debreu's result to generalized abstract economy without order preferences. Then, the result was extended to many directions, see, for example, [3-9] and the references therein.

As is known to all, Zadeh [10] established the fuzzy set theory to treat the scenarios which cannot be characterized precisely as an attempt to develop the mathematical framework. Since then, the theory of fuzzy sets has been extended to many areas. Butnariu [11] first established the characterization of the fuzzy game. Billot [4] obtained some equilibrium existence theorems of fuzzy games. Huang $[12,13]$ studied a new class of generalized abstract fuzzy economies with uncountable number of players and proved some new existence theorems of maximal element for the abstract fuzzy economies and qualitative fuzzy games, respectively. For more about the fuzzy games and fuzzy abstract economies, we refer to [14-18].

On the other hand, we know that both the Nash equilibrium and the Debreu equilibrium are established on the assumption of perfect rationality of the players in the game. This assumption in the economic models is too strict. Ideally, we would like to build a model of bounded rationality that could be meaningful not only in theory but also in application. For this purpose, Anderlini and Canning [19] established the abstract framework, a model $M$, that consists of a parameterized class of general games and an associated abstract rationality function. They discussed the relationship of the robustness to bounded rationality and structural stability for the model $M$. They also applied the result to four economic examples. Under the weak assumptions of Anderlini and Canning [19], C. Yu and J. Yu [20, 21] proved the following result: the model $M$ is structurally stable and robust to $\epsilon$-equilibria for almost all parameter values of generalized games and multiobjective games. Recently, Wang et al. [22] improved the economic model proposed by Anderlini and Canning, and under the weaker assumptions, they also showed that the model $M$ is structurally stable if and only if it is robust to $\epsilon$-equilibria in generalized convex spaces. Miyazaki and Azuma [23] generalize the definitions of structurally stable and robustness to $\epsilon$-equilibria that are introduced in Anderlini and Canning [19] and C. Yu and J. $\mathrm{Yu}[20,21]$. They also studied the essentiality of equilibria in a bounded rational environment.

Motivated and inspired by the work mentioned above, in this paper, a new class of generalized abstract fuzzy economies with an uncountable number of agents with fuzzy constraint correspondences and fuzzy preference correspondence in finite continuous spaces without any convexity structure is established. Then, a new existence theorem for generalized abstract fuzzy economies with an uncountable 
number of agents with fuzzy constraint correspondences and fuzzy preference correspondence in locally finite continuous uniform spaces is established in Section 3. Finally, applying the result in Section 3, we obtain that the model $M$, which consists of a parameterized class of generalized abstract fuzzy economies and the associated abstract rationality function, is structurally stable at $(\lambda, \epsilon)$ if and only if $M$ is robust to $(\lambda, \epsilon)$ equilibria in Section 4 . The results presented in this paper generalize some known results in [12, 13, 19-22].

\section{Preliminaries}

Let $2^{X}$ and $\langle X\rangle$ be the family of all subsets and the family of all nonempty finite subsets of a set $X$, respectively. Let $|A|$ be the cardinality of $A$, for arbitrary $A \in\langle X\rangle$. We denote by $\Delta_{n}$ the standard $n$-dimensional simplex with vertices $e_{0}, e_{1}, \ldots, e_{n}$. For any nonempty set $J$ of $\{0,1, \ldots, n\}$, we denote by $\Delta_{J}$ the convex hull of the vertices $\left\{e_{j}: j \in J\right\}$. Ding and Wang [7] first introduced the following definition of finitely continuous topological space (or FC-space) without any convexity structure.

Definition 1. A finitely continuous space (for short, FC-space) $\left(X,\left\{\varphi_{N}\right\}\right)$ consists of a topological space $X$ and a mapping $\varphi_{N}$ such that for each $N=\left\{x_{0}, \ldots, x_{n}\right\} \in\langle X\rangle$, where some elements in $N$ may be the same, there exists a continuous mapping $\varphi_{N}: \Delta_{n} \rightarrow X$. For each $N=\left\{x_{0}, \ldots, x_{n}\right\} \in\langle X\rangle$ and for any $\left\{x_{i 0}, \ldots, x_{i k}\right\} \subset B \cap N$, if $\varphi_{N}\left(\Delta_{k}\right) \subset D$, then a subset $B$ of $\left(X,\left\{\varphi_{N}\right\}\right)$ is said to be an FC-subspace of $X$.

It is easy to see that each FC-subspace of $\left(X,\left\{\varphi_{N}\right\}\right)$ is also a FC-space by the definition of FC-subspaces of a FC-space, and if $\left\{D_{i}\right\}_{i \in I}$ is a family of FC-subspaces of an FC-space $\left(X,\left\{\varphi_{N}\right\}\right)$ and $\bigcap_{i \in I} B_{i} \neq \emptyset$, then $\bigcap_{i \in I} D_{i}$ is also an FC-subspace of $\left(X,\left\{\varphi_{N}\right\}\right)$, where $I$ is any index set.

Definition 2 (see [24]). If $(X, \mathcal{U})$ is a uniform space and $\left(X,\left\{\varphi_{N}\right\}\right)$ is an FC-space such that $\mathcal{U}$ has a basis $\mathscr{B}$ consisting of entourages satisfying that for each $V \in \mathscr{B}$, the set $\{x \in X$ : $M \cap V[x] \neq \emptyset\}$ is an FC-subspace of $X$ whenever $M \in X$ is an FC-subspace of $X$, and then $\left(X, \mathcal{U},\left\{\varphi_{N}\right\}\right)$ is said to be a locally FC-uniform space.

We denote by $X$ and $Y$ both metric spaces as follows.

(i) If $G(X)$ is contained in some compact subset of $Y$, then a set-valued mapping $G: X \rightarrow 2^{Y}$ is said to be compact.

(ii) If for each $x \in X$ and for each open set $U$ in $Y$, the set $\{x \in X: G(x) \subseteq U\}$ is open in $X$, then $G$ is said to be upper semicontinuous on $X$.

(iii) If for each $x \in X$ and for each open set $U$ in $Y$, the set $\{x \in X: G(x) \cap U \neq \emptyset\}$ is open in $X$, then $G$ is said to be lower semicontinuous on $X$.

(iv) If $G$ is both upper semicontinuous and lower semicontinuous on $X$, then $G$ is said to be continuous on $X$.

Furthermore, if $Y$ is a compact metric space, then (i) $G$ is upper semicontinuous at $x \in X$ if and only if for any $x_{n} \rightarrow x$ and $y_{n} \in G\left(x_{n}\right), y_{n} \rightarrow y$; then, $y \in G(x)$;

(ii) $G$ is lower semicontinuous at $x$ if and only if for any $x_{n} \rightarrow x$ and $y \in G(x)$, there exists $y_{n} \in G\left(x_{n}\right), y_{n} \rightarrow$ $y$;

(iii) $G$ is continuous at $x$ if and only if for any $x_{n} \rightarrow x$, $h\left(G\left(x_{n}\right), G(x)\right) \rightarrow 0$, where $h$ is the Hausdorff distance defined on $Y$.

\section{Equilibria of Generalized Abstract Fuzzy Economies}

In this section, a new existence theorem for generalized abstract fuzzy economies with an uncountable number of agents with fuzzy constraint correspondences and fuzzy preference correspondence in locally FC-uniform spaces is established.

Throughout this paper, let $\mathscr{F}(X)(\mathscr{F}(Y))$ be the collection of all fuzzy sets on $X(Y)$, where $M$ and $N$ are two Hausdorff topological vector spaces and $X \subset M$ and $Y \subset N$ are two nonempty convex subsets. We call a mapping from $X$ to $\mathscr{F}(Y)(\mathscr{F}(X))$ a fuzzy mapping, and then for each $x \in X$, $F(x)$ (denote by $F_{x}$ ) is a fuzzy set in $\mathscr{F}(Y)$ and $F_{x}(y)$ is the degree of membership of point $y$ in $F_{x}$.

If for each $x \in X$, a fuzzy set $F_{x}$ on $Y$ is a fuzzy convex set, then a fuzzy mapping $F: X \rightarrow \mathscr{F}(Y)$ is called convex; that is,

$$
F_{x}\left(k y_{1}+(1-k) y_{2}\right) \geq \min \left\{F_{x}\left(y_{1}\right), F_{x}\left(y_{2}\right)\right\}
$$

for any $y_{1}, y_{2} \in Y, k \in[0,1]$.

For any $q \in[0,1]$, we denote by $(A)_{q}=\{x \in X: A(x) \geq$ q) the $q$-cut set of $A \in \mathscr{F}(X)$.

A generalized abstract fuzzy economy $\Gamma=\left(X_{i}, A_{i}\right.$, $\left.B_{i}, P_{i}\right)_{i \in I}$ consists of a nonempty topological space (a choice set) $X_{i}$, fuzzy constraint mappings (fuzzy constraint correspondences) $A_{i}, B_{i}: X=\prod_{i \in I} X_{i} \rightarrow \mathscr{F}\left(X_{i}\right)$, a fuzzy preference mapping (fuzzy preference correspondence) $P_{i}: X \rightarrow$ $\mathscr{F}\left(X_{i}\right)$, and a finite or an infinite set of agents $I$. A point $\widehat{x} \in X$ is said to be an equilibrium for $\Gamma$ if each $i \in I, \widehat{x}_{i} \in\left(B_{i \widehat{x}}\right)_{b_{i}(\widehat{x})}$ and $\left(A_{i \widehat{x}}\right)_{a_{i}(\widehat{x})} \cap\left(P_{i \widehat{x}}\right)_{p_{i}(\widehat{x})}=\emptyset$, where $a_{i}, b_{i}, p_{i}: X \rightarrow(0,1]$.

Theorem 3. Let $\left(X_{i}, A_{i}, B_{i}, P_{i}\right)_{i \in I}$ be an abstract fuzzy economy, $X=\prod_{i \in I} X_{i}$, and $a_{i}, b_{i}, p_{i}: X \rightarrow(0,1]$ such that for each $i \in I$, the following conditions are satisfied:

(1) $\left(X_{i}, \mathscr{U}_{i},\left\{\varphi_{N_{i}}\right\}\right)$ is a compact locally FC-uniform space;

(2) for each $x \in X, x \rightarrow\left(A_{i x}\right)_{a_{i}(x)}: X \rightarrow 2^{X_{i}}$ and $x \rightarrow$ $\left(B_{i x}\right)_{b_{i}(x)}: X \rightarrow 2^{X_{i}}$ are both upper semicontinuous compact mappings with nonempty closed values;

(3) for each $x \in X,\left(A_{i x}\right)_{a_{i}(x)}$ and $\left(B_{i x}\right)_{b_{i}(x)}$ are nonempty FC-subspaces of $X_{i}$;

(4) for each $x \in X, x \rightarrow\left(P_{i x}\right)_{p_{i}(x)}: X \rightarrow 2^{X_{i}}$ is a closed mapping such that for each $x \in X,\left(P_{i x}\right)_{p_{i}(x)}$ is an FCsubspace of $X_{i}$;

(5) the set $E_{i}=\left\{x \in X:\left(A_{i x}\right)_{a_{i}(x)} \cap\left(P_{i x}\right)_{p_{i}(x)} \neq \emptyset\right\}$ is open in $X$; 
(6) for each $x \in X, x_{i} \notin\left(A_{i x}\right)_{a_{i}(x)} \cap\left(P_{i x}\right)_{p_{i}(x)}$.

Then, there exists a point $\widehat{x} \in X$ such that for each $i \in I, \widehat{x}_{i} \in$ $\left(B_{i \widehat{x}}\right)_{b_{i}(\widehat{x})}$ and

$$
\left(A_{i \widehat{x}}\right)_{a_{i}(\widehat{x})} \cap\left(P_{i \widehat{x}}\right)_{p_{i}(\widehat{x})}=\emptyset .
$$

Proof. It is easy to see that $\left(X, \mathcal{U},\left\{\varphi_{N}\right\}\right)$ becomes a locally FCuniform space by Lemma 2.1 in [24]. For each $i \in I$, define the following correspondence $G_{i}: X \rightarrow 2^{X_{i}}$ by

$$
G_{i}(x)= \begin{cases}\left(A_{i x}\right)_{a_{i}(x)} \cap\left(P_{i x}\right)_{p_{i}(x)}, & \text { if } x \in E_{i}, \\ \left(B_{i x}\right)_{b_{i}(x)}, & \text { if } x \notin E_{i} .\end{cases}
$$

Next, we will show that the family $\left\{G_{i}\right\}$ is an upper semicontinuous compact mapping with nonempty compact values. First, for each $x \in X, G_{i}(x)$ is a nonempty FC-subspace of $X_{i} ; x \rightarrow\left(A_{i x}\right)_{a_{i}(x)} \cap\left(P_{i x}\right)_{p_{i}(x)}$ is an upper semicontinuous compact mapping on $X$ with nonempty closed values because of the conditions (3) and (4). On the other hand, by the conditions (2)-(5), Lemma 3 of Fan [25], and Lemma 3.1 of [26], $G_{i}$ is an upper semicontinuous compact mapping with nonempty closed values. Now, we define a set-valued mapping $G: X \rightarrow 2^{X}$ by

$$
G(x)=\prod_{i \in I} G_{i}(x), \quad \forall x \in X .
$$

For each $i \in I, G_{i}$ is an upper semicontinuous compact mapping with nonempty compact values such that for each $x \in X, G_{i}(x)$ is an FC-subspace of $X_{i}$. By Lemma 3 of Fan [25] and Lemma 2.1 of [24], $G$ is also upper semicontinuous compact mapping with nonempty closed values such that for each $x \in X, G(x)$ is an FC-subspace of $X$. From Lemma 2.2 of [24], we know that there exists a point $\widehat{x} \in X$ such that $\hat{x} \in G(\hat{x})$; that is, $\hat{x}_{i} \in G_{i}(\hat{x})$ for each $i \in I$. If for some $j \in I, \hat{x} \in E_{j}$, then we have $\widehat{x}_{j} \in\left(A_{j \hat{x}}\right)_{a_{j}(\hat{x})} \cap\left(P_{j \hat{x}}\right)_{p_{j}(\hat{x})}$ which contradicts the condition (6). Therefore, we must have that $\widehat{x} \notin E_{i}$ for all $i \in I$. It follows from the definition of $G_{i}$ that for each $i \in I, \widehat{x}_{i} \in\left(B_{i \widehat{x}}\right)_{b_{i}(\widehat{x})}$ and $\left(A_{i \widehat{x}}\right)_{a_{i}(\widehat{x})} \cap\left(P_{i \widehat{x}}\right)_{p_{i}(\widehat{x})}=\emptyset$. This completes the proof of Theorem 3 .

Remark 4. Theorem 3 extends and improves Theorem 3.1 of Huang [8], Theorem 3.1 of Huang [12], and Lemma 3.1 of Wang et al. [22] in the following aspects: (1) the generalized abstract fuzzy economy model we considered in Theorem 3 is more general; (2) we generalize the related results from the convex subset of a topological vector space and the generalized convex space to FC-space without any convexity structure. Theorem 3 is also an improved variant of Theorem 3.1 of Ding [24] in locally FC-uniform spaces. Finally, we emphasize that, FC-spaces include many topological spaces as special cases, for example, the convex subset of a topological vector space and the generalized convex space that were just mentioned and the $H$-space.

\section{Structural Stability and Robustness to $(\lambda, \epsilon)$-Equilibria}

In this section, firstly, applying the result in Section 3, we study the model $M$, which consists of a parameterized class of generalized abstract fuzzy economies and the associated abstract rationality function. Then, we obtain that the model $M$ is structurally stable at $(\lambda, \epsilon)$ if and only if $M$ is robust to $(\lambda, \epsilon)$-equilibria.

Let $\Gamma=\left(X_{i}, A_{i}, B_{i}, P_{i}\right)_{i \in I}$ be a generalized abstract fuzzy economy and satisfy all the conditions of Theorem 3 . Then, from Theorem 3, we know that

$$
G_{i}(x)= \begin{cases}\left(A_{i x}\right)_{a_{i}(x)} \cap\left(P_{i x}\right)_{p_{i}(x)}, & \text { if } x \in E_{i}, \\ \left(B_{i x}\right)_{b_{i}(x)}, & \text { if } x \notin E_{i}\end{cases}
$$

is an upper semicontinuous compact mapping on $X=$ $\prod_{i \in I} X_{i}$. Now we define that $\Lambda=\left\{\lambda=\left\{G_{i}\right\}_{i \in I}: G_{i}: X=\prod_{i \in I}\right.$ $\left.X_{i} \rightarrow 2^{X_{i}}\right\}$. By the conclusion from Theorem 3 , we have that there exists a point $\widehat{x} \in X$ such that $\widehat{x}_{i} \in G_{i}(\hat{x})$, for each $i \in I$, and $\widehat{x}$ is an equilibrium point of the generalized abstract fuzzy economy $\Gamma$. We denote by $E(\lambda)$ the set of all equilibrium points of generalized abstract fuzzy economy $\Gamma$ for each $\lambda \in \Lambda$. By Theorem 3 , we know that $E(\lambda) \neq \emptyset$.

Let $X_{i}$ be a metric space. For any $\lambda_{1}=\left\{G_{1 i}\right\}_{i \in I} \in \Lambda$ and $\lambda_{2}=\left\{G_{2 i}\right\}_{i \in I} \in \Lambda$, we define that

$$
\rho\left(\lambda_{1}, \lambda_{2}\right)=\sum_{i \in I} \sup _{x \in X} h_{i}\left(G_{1 i}(x), G_{2 i}(x)\right),
$$

where $h_{i}$ is the Hausdorff metric induced on $X_{i}$ induced by a metric $d_{i}$ for each $i \in I$.

Lemma 5. $(\Lambda, \rho)$ is a complete metric space.

Proof. We denote by $\left\{\lambda_{m}\right\}$ any Cauchy sequence in $\Lambda$. Then, for any $\epsilon>0$, there exists a positive integer $P(\epsilon)$ such that $\forall m, p \geq P(\epsilon)$; we have

$$
\rho\left(\lambda_{m}, \lambda_{p}\right)=\sum_{i \in I} \sup _{x \in X} h_{i}\left(G_{m i}(x), G_{p i}(x)\right)<\epsilon .
$$

It is easy to see that $X_{i}$ is complete and $X=\prod_{i \in I} X_{i}$ is compact by the compactness of $X_{i}$. On the other hand, under the Hausdorff distance, the family of compact subsets of $X_{i}$ is a complete metric space. Since $G_{m i}: X \rightarrow 2^{X_{i}}$ is a upper semicontinuous compact mapping on $X$, by Proposition 3.1.11 in [27], we have that there exists a compact set $G_{i}(x) \subset X_{i}$, such that $\lim _{p \rightarrow \infty} G_{p i}(x)=G_{i}(x)$, for all $i \in I$. It follows from (7) that $\forall m \geq P(\epsilon)$,

$$
\sum_{i \in I} \sup _{x \in X} h_{i}\left(G_{m i}(x), G_{i}(x)\right) \leq \epsilon .
$$

Because of $\lambda_{m}=\left\{G_{m i}\right\}_{i \in I} \in \Lambda$, there exists $x_{m} \in X$, such that $x_{m i} \in G_{m i}\left(x_{m}\right)$, for each $i \in I$. Without loss of generality, applying the conclusion mentioned above that $X$ is compact, let $x_{m} \rightarrow x$. It is easy to check that since $G_{i}$ is upper semicontinuous on $X$, there exists a positive integer $m_{0} \geq P(\epsilon)$ such that, $\forall m \geq m_{0}$,

$$
G_{i}\left(x_{m}\right) \subset U\left(\epsilon, G_{i}(x)\right) .
$$

Then, we have $\forall m \geq m_{0}$,

$$
x_{m i} \in G_{m i}\left(x_{m}\right) \subset U\left(2 \epsilon, G_{i}\left(x_{m}\right)\right) \subset U\left(3 \epsilon, G_{i}(x)\right) .
$$


Let $m \rightarrow \infty$; we have that $d_{i}\left(x_{i}, G_{i}(x)\right) \leq 3 \epsilon$. Since $\epsilon$ can be arbitrary small, we get $d_{i}\left(x_{i}, G_{i}(x)\right)=0$ and $x_{i} \in G_{i}(x)$. Then, we have $\lambda=\left\{G_{i}\right\}_{i \in I} \in \Lambda$. Therefore, $(\Lambda, \rho)$ is a complete metric space. This completes the proof.

Now, we consider the model $M=(\Lambda, X, F, R)$, where

(i) $\Lambda$ is a complete metric space;

(ii) $X$ is a metric space;

(iii) for any $x \in X$ and for all $\lambda=\left\{G_{i}\right\}_{i \in I} \in \Lambda$, define $F(\lambda, x)=G(x)=\prod_{i \in I} G_{i}(x)$. For all $\lambda \in \Lambda$, the continuous correspondence $f: \Lambda \rightarrow 2^{X}$ denoted by $f(\lambda)=\{x \in X: x \in F(\lambda, x)\}=\left\{x \in X: x_{i} \in G_{i}(x)\right\}$ and for all $\lambda \in \Lambda, f(\lambda)$ is a nonempty compact;

(iv) for any $x \in f(\lambda)$, define $R$ : Gragh $(f) \rightarrow R^{+}$by

$$
R(\lambda, x)=\max _{i \in I} d_{i}\left(x_{i}, G_{i}(x)\right)=\max _{i \in I} \min _{y_{i} \in G_{i}(x)} d_{i}\left(x_{i}, y_{i}\right),
$$

where $d_{i}$ is the distance on $X_{i}$, for each $i \in I$.

$R:$ Gragh $(f) \rightarrow R^{+}$is a rationality function, and $R(\lambda$, $x)=0$ means the full rationality.

For any $\lambda \epsilon \Lambda$ and any $\epsilon \geq 0$, we define the set of $\epsilon$ equilibria of generalized abstract fuzzy economy $\Gamma$ at $\lambda$ by

$$
E(\lambda, \epsilon)=\{x \in f(\lambda): R(\lambda, x) \leq \epsilon\} .
$$

As a special case, we define the set of all equilibrium points of generalized abstract fuzzy economy $\Gamma$ at $\lambda$ by

$$
E(\lambda)=E(\lambda, 0)=\{x \in f(\lambda): R(\lambda, x)=0\} .
$$

It is easy to see that for all $(\lambda, \epsilon) \in \Lambda \times R^{+}$, if $R$ is lower semicontinuous, then $E(\lambda, \epsilon)$ is compact.

Note that $R(\lambda, x) \geq 0$ and $R(\lambda, x)=0$ if and only if $x_{i} \in$ $G_{i}(x)$ and $x \in E(\lambda)$ for every $i \in I$.

Lemma 6. $R(\lambda, x)$ is lower semicontinuous at $(\lambda, x)$.

Proof. First, we need to prove that $\forall \lambda_{n}=\left\{G_{n i}\right\}_{i \in I} \in \Lambda$ with $\rho\left(\lambda_{n}, \lambda\right) \rightarrow 0$, where $\lambda=\left\{G_{i}\right\}_{i \in I} \in \Lambda$ and $\forall x_{n} \in X$ with $x_{n} \rightarrow x$; the following inequality holds:

$$
R\left(\lambda_{n}, x_{n}\right)>R(\lambda, x)-\epsilon, \quad \forall \epsilon>0 .
$$

It is easy to see that $G_{i}: X \rightarrow 2^{X_{i}}$ is compact upper semicontinuous, and from Proposition 3.1.19 in [27], we obtain that $d_{i}\left(x_{i}, G_{i}(x)\right)=\min _{y_{i} \in G_{i}(x)} d_{i}\left(x_{i}, y_{i}\right)$ is lower semicontinuous at $x_{i}$. Thus, we get $\exists N_{1}$, where $N_{1}$ is a positive integer, such that, $\forall n \geq N_{1}$,

$$
\min _{y_{i} \in G_{i}\left(x_{n}\right)} d_{i}\left(x_{n i}, y_{i}\right)>\min _{y_{i} \in G_{i}(x)} d_{i}\left(x_{i}, y_{i}\right)-\frac{\epsilon}{2}, \quad \forall i \in I
$$

Since for any $n=1,2, \ldots, G_{n i}$ is compact, we get that $\exists y_{n i} \in$ $G_{n i}\left(x_{n}\right)$ such that

$$
d_{i}\left(x_{n i}, y_{n i}\right)=\min _{y_{i} \in G_{n i}\left(x_{n}\right)} d_{i}\left(x_{n i}, y_{i}\right), \quad \forall i \in I
$$

On the other hand, because of $h_{i}\left(G_{n i}\left(x_{n}\right), G_{i}\left(x_{n}\right)\right) \rightarrow 0$ and $y_{n i} \in G_{n i}\left(x_{n}\right)$, we know that there exists a positive integer $N_{2}$ such that, $\forall n \geq N_{2}, \exists y_{n i}^{\prime} \in G_{i}\left(x_{n}\right)$ with $d\left(y_{n i}^{\prime}, y_{n i}\right)<\epsilon / 2$ for each $i \in I$. Letting $N=\max \left\{N_{1}, N_{2}\right\}, \forall n \in N$, we obtain that

$$
\begin{aligned}
R\left(\lambda_{n}, x_{n}\right) & =\max _{i \in I} \min _{y_{i} \in G_{n i}\left(x_{n}\right)} d_{i}\left(x_{n i}, y_{i}\right) \\
& =\max _{i \in I} d_{i}\left(x_{n i}, y_{n i}\right) \\
& \geq \max _{i \in I}\left(d_{i}\left(x_{n i}, y_{n i}^{\prime}\right)-d_{i}\left(y_{n i}^{\prime}, y_{n i}\right)\right) \\
& \geq \max _{i \in I} d_{i}\left(x_{n i}, y_{n i}^{\prime}\right)-\max _{i \in I} d_{i}\left(y_{n i}^{\prime}, y_{n i}\right) \\
& \geq \max _{i \in I} \min _{y_{i} \in G_{i}\left(x_{n}\right)} d_{i}\left(x_{n i}, y_{i}\right)-\frac{\epsilon}{2} \\
& >\max _{i \in I} \min _{y_{i} \in G_{i}(x)} d_{i}\left(x_{i}, y_{i}\right)-\epsilon \\
& =R(\lambda, x)-\epsilon .
\end{aligned}
$$

Now, we obtain the conclusion that $R(\lambda, x)$ is lower semicontinuous at $(\lambda, x)$. This completes the proof.

The following concepts of robust to $(\lambda, \epsilon)$-equilibria and structurally stable at $(\lambda, \epsilon)$ were introduced by Miyazaki and Azuma [23].

Definition 7. If $\forall \delta>0$, there exists $\bar{\epsilon}>0$ such that, $\forall \epsilon^{\prime}>0$ with $\left|\epsilon-\epsilon^{\prime}\right|<\bar{\epsilon}$ and $\forall \lambda^{\prime} \in \Lambda$ with $d\left(\lambda, \lambda^{\prime}\right)<\bar{\epsilon}$ and $h\left(E\left(\lambda^{\prime}, \epsilon\right), E\left(\lambda^{\prime}, \epsilon^{\prime}\right)\right)<\delta$; the model $M$ is said to be robust to $(\lambda, \epsilon)$-equilibria, where $h$ is the Hausdorff distance defined on $X$.

Definition 8. If the bounded rational equilibrium correspondence $E: \Lambda \times R^{+} \rightarrow 2^{X}$ is continuous at $(\lambda, \epsilon)$, the model $M$ is said to be structurally stable at $(\lambda, \epsilon)$.

Theorem 9. Under the assumptions of model $M$, then, $M$ is structurally stable at $(\lambda, \epsilon)$ which implies that $M$ is robust to $(\lambda, \epsilon)$-equilibria.

Proof. Suppose that $M$ is not robust to $(\lambda, \epsilon)$-equilibria. Then, there are $\delta \geq 0$, sequence $\left\{\epsilon_{n}\right\}_{n \in N}$ with $\epsilon_{n}>0$ for all $n \in N$ and $\epsilon_{n} \rightarrow \epsilon$, and $\lambda_{n} \rightarrow \lambda$ such that

$$
h\left(E\left(\lambda_{n}, \epsilon_{n}\right), E\left(\lambda_{n}, \epsilon\right)\right) \geq \delta .
$$

Because of $E\left(\lambda_{n}, \epsilon\right) \subset E\left(\lambda_{n}, \epsilon_{n}\right)$, we can choose $x_{n} \in E\left(\lambda_{n}, \epsilon_{n}\right)$ such that

$$
\min _{v \in E\left(\lambda_{n}, \epsilon\right)} d\left(x_{n}, v\right)>\frac{\delta}{2} .
$$

Let $x_{n} \rightarrow x$, because $X$ is compact. Since $E: \Lambda \times R^{+} \rightarrow 2^{X}$ is continuous at $(\lambda, \epsilon)$, we get that $h\left(E\left(\lambda_{n}, \epsilon_{n}\right), E(\lambda, \epsilon)\right) \rightarrow 0$. By the Lemma 3.2 in [20], we obtain that

$$
\min _{v \in E\left(\lambda_{n}, \epsilon\right)} d\left(x_{n}, v\right) \geq \frac{\delta}{2} .
$$


On the other hand, since

$$
f(\lambda)=\{x \in X: x \in F(\lambda, x)\}=\left\{x \in X: x_{i} \in G_{i}(x)\right\}
$$

we obtain that $f: \Lambda \rightarrow 2^{X}$ is upper semicontinuous from Lemma 6 in [28]. Since $x_{n} \in f\left(\lambda_{n}\right)$, then $x \in f(\lambda)$. Since $R$ is lower semicontinuous and $R\left(\lambda_{n}, x_{n}\right) \leq \epsilon_{n}$, then $R(\lambda, x) \leq$ $\lim _{n \rightarrow \infty} R\left(\lambda_{n}, x_{n}\right)=0$. Thus we obtain $x \in E(\lambda, \epsilon)$ which contradicts that $\min _{v \in E\left(\lambda_{n}, \epsilon\right)} d\left(x_{n}, v\right) \geq \delta / 2$. Thus, we have that $M$ is robust to $(\lambda, \epsilon)$-equilibria. This completes the proof.

Remark 10. Theorem 9 improves and generalizes Theorem 3.1 of [19] in the following aspects: (1) $\Lambda$ is a complete metric space, $X$ is a metric space, and we dropped the compactness of $\Lambda$; (2) the continuity of $f$ is weakened to the upper semicontinuity; (3) the continuity of $F$ is weakened to the lower semicontinuity. Compared with Theorem 3.1 of C. Yu and J. Yu [20, 21] and Theorem 3.1 of Wang et al. [22], the model we considered in Theorem 9 is more general and we generalize the related conclusions from structural stability at $\lambda$ to structural stability at $(\lambda, \epsilon)$ and from robustness to $\epsilon$ equilibria to robustness to $(\lambda, \epsilon)$-equilibria.

\section{Conflict of Interests}

The authors declare that there is no conflict of interests regarding the publication of this paper.

\section{Acknowledgments}

This work was supported by the National Natural Science Foundation of China $(11126346,11201379)$ and the Fundamental Research Funds for the Central Universities of China (JBK130401).

\section{References}

[1] K. J. Arrow and G. Debreu, "Existence of an equilibrium for a competitive economy," Econometrica, vol. 22, pp. 265-290, 1954.

[2] A. Borglin and H. Keiding, "Existence of equilibrium actions and of equilibrium: a note on the "new" existence theorems," Journal of Mathematical Economics, vol. 3, no. 3, pp. 313-316, 1976.

[3] Q. H. Ansari and J.-C. Yao, "An existence result for the generalized vector equilibrium problem," Applied Mathematics Letters, vol. 12, no. 8, pp. 53-56, 1999.

[4] A. Billot, Economic Theory of Fuzzy Equilibria, vol. 373 of Lecture Notes in Economics and Mathematical Systems, Springer, Berlin, Germany, 1992.

[5] W. Briec and C. Horvath, "Nash points, Ky Fan inequality and equilibria of abstract economies in Max-Plus and B-convexity," Journal of Mathematical Analysis and Applications, vol. 341, no. 1, pp. 188-199, 2008.

[6] L.-C. Ceng, Q. H. Ansari, and J.-C. Yao, "Hybrid pseudoviscosity approximation schemes for equilibrium problems and fixed point problems of infinitely many nonexpansive mappings," Nonlinear Analysis: Hybrid Systems, vol. 4, no. 4, pp. 743-754, 2010.
[7] X. P. Ding and L. Wang, "Fixed points, minimax inequalities and equilibria of noncompact abstract economies in FC-spaces," Nonlinear Analysis: Theory, Methods \& Applications, vol. 69, no. 2, pp. 730-746, 2008.

[8] N. Huang, "Some new equilibrium theorems for abstract economies," Applied Mathematics Letters, vol. 11, no. 1, pp. 4145, 1998.

[9] L.-J. Lin, L.-F. Chen, and Q. H. Ansari, "Generalized abstract economy and systems of generalized vector quasi-equilibrium problems," Journal of Computational and Applied Mathematics, vol. 208, no. 2, pp. 341-353, 2007.

[10] L. A. Zadeh, "Fuzzy sets," Information and Control, vol. 8, no. 3, pp. 338-353, 1965.

[11] D. Butnariu, "Fuzzy games: a description of the concept," Fuzzy Sets and Systems, vol. 1, no. 3, pp. 181-192, 1978.

[12] N.-J. Huang, "A new equilibrium existence theorem for abstract fuzzy economies," Applied Mathematics Letters, vol. 12, no. 5, pp. $1-5,1999$.

[13] N.-J. Huang, "Existence of equilibrium for generalized abstract fuzzy economies," Fuzzy Sets and Systems, vol. 117, no. 1, pp. 151156, 2001.

[14] Y. Azrieli and E. Lehrer, "On some families of cooperative fuzzy games," International Journal of Game Theory, vol. 36, no. 1, pp. $1-15,2007$.

[15] S. Borkotokey, "Cooperative games with fuzzy coalitions and fuzzy characteristic functions," Fuzzy Sets and Systems, vol. 159, no. 2, pp. 138-151, 2008.

[16] Y.-A. Hwang and Y.-H. Liao, "The consistent value of fuzzy games," Fuzzy Sets and Systems, vol. 160, no. 5, pp. 644-656, 2009.

[17] W. K. Kim and K. H. Lee, "Fuzzy fixed point and existence of equilibria in fuzzy games," Journal of Fuzzy Mathematics, vol. 6, no. 1, pp. 193-202, 1998.

[18] S. Li and Q. Zhang, "A simplified expression of the Shapley function for fuzzy game," European Journal of Operational Research, vol. 196, no. 1, pp. 234-245, 2009.

[19] L. Anderlini and D. Canning, "Structural stability implies robustness to bounded rationality," Journal of Economic Theory, vol. 101, no. 2, pp. 395-422, 2001.

[20] C. Yu and J. Yu, "Bounded rationality in multiobjective games," Nonlinear Analysis: Theory, Methods \& Applications, vol. 67, no. 3, pp. 930-937, 2007.

[21] C. Yu and J. Yu, "On structural stability and robustness to bounded rationality," Nonlinear Analysis: Theory, Methods \& Applications, vol. 65, no. 3, pp. 583-592, 2006.

[22] L. Wang, Y. J. Cho, and N.-J. Huang, "The robustness of generalized abstract fuzzy economies in generalized convex spaces," Fuzzy Sets and Systems, vol. 176, pp. 56-63, 2011.

[23] Y. Miyazaki and H. Azuma, " $(\lambda, \epsilon)$-stable model and essential equilibria," Mathematical Social Sciences, vol. 65, no. 2, pp. 8591, 2013.

[24] X. P. Ding, “The generalized game and the system of generalized vector quasi-equilibrium problems in locally $F C$-uniform spaces," Nonlinear Analysis: Theory, Methods \& Applications, vol. 68, no. 4, pp. 1028-1036, 2008.

[25] Ky. Fan, "Fixed-point and minimax theorems in locally convex topological linear spaces," Proceedings of the National Academy of Sciences of the United States of America, vol. 38, pp. 121-126, 1952.

[26] G. X.-Z. Yuan, KKM Theory and Applications in Nonlinear Analysis, vol. 218 of Monographs and Textbooks in Pure and Applied Mathematics, Marcel Dekker, New York, NY, USA, 1999. 
[27] J.-P. Aubin and I. Ekeland, Applied Nonlinear Analysis, John Wiley \& Sons, New York, NY, USA, 1984.

[28] K.-K. Tan, J. Yu, and X.-Z. Yuan, "The stability of coincident points for multivalued mappings," Nonlinear Analysis: Theory, Methods \& Applications, vol. 25, no. 2, pp. 163-168, 1995. 


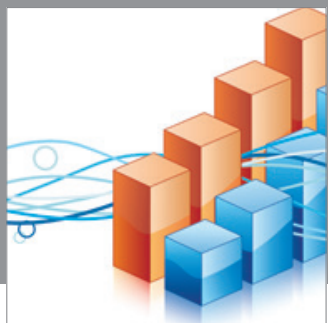

Advances in

Operations Research

mansans

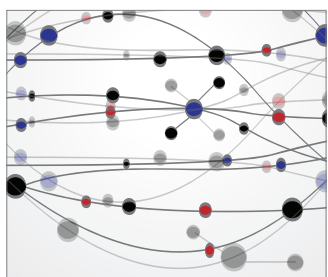

The Scientific World Journal
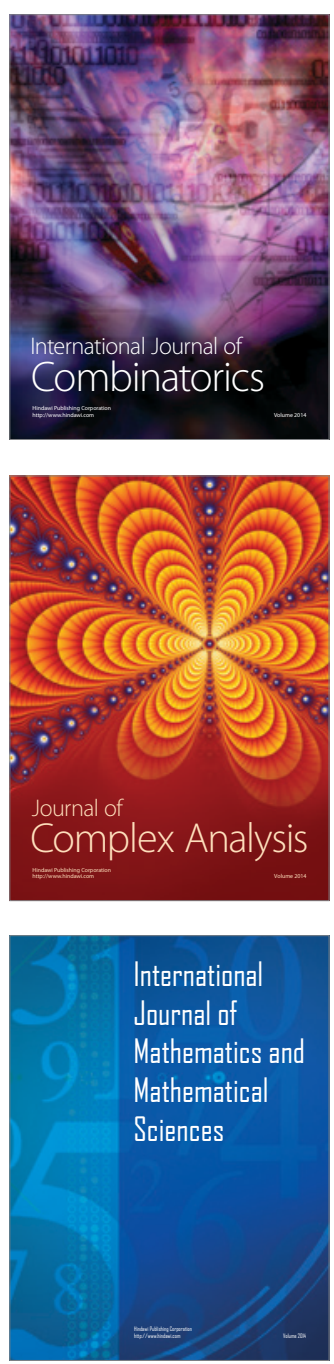
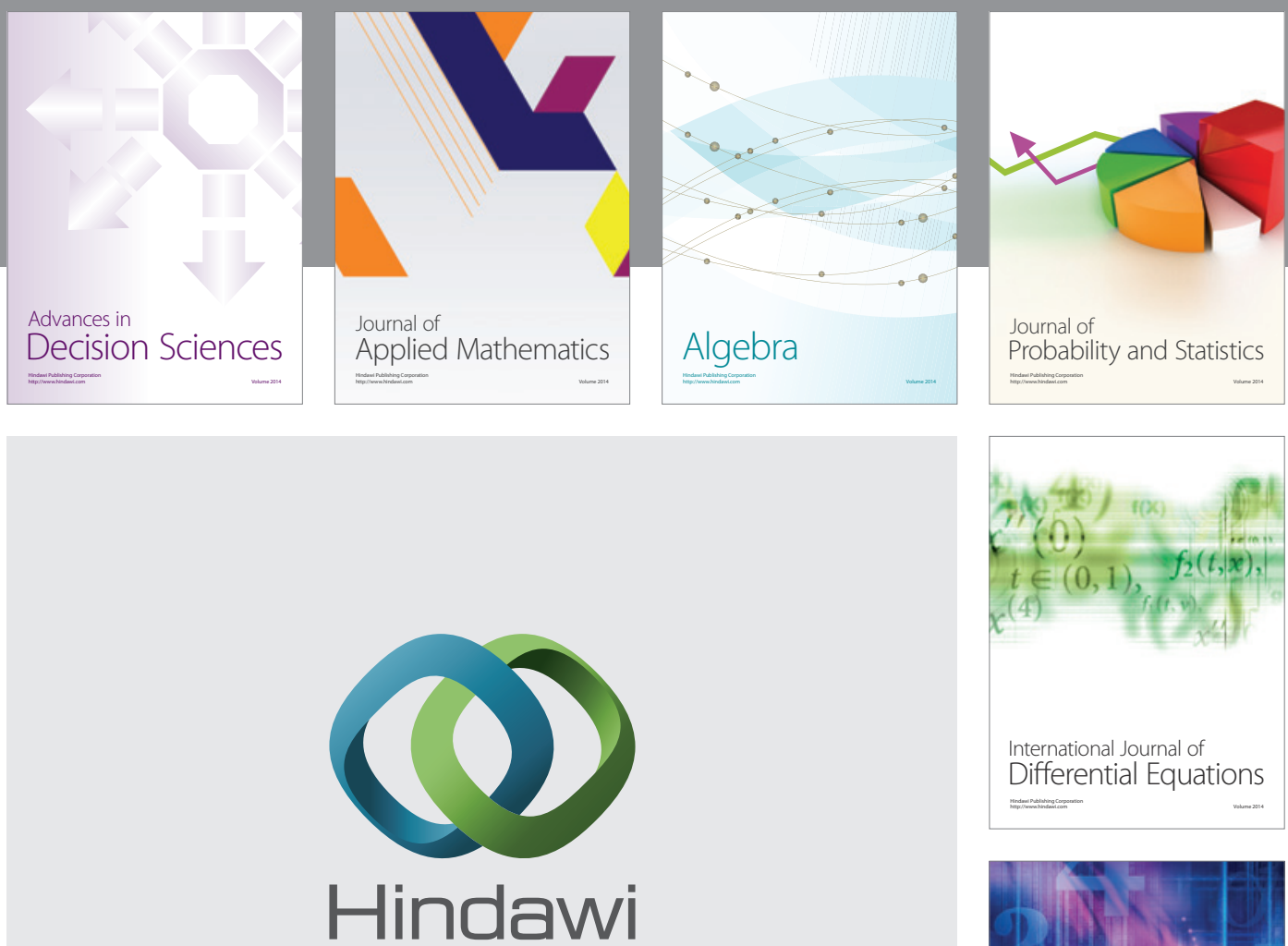

Submit your manuscripts at http://www.hindawi.com
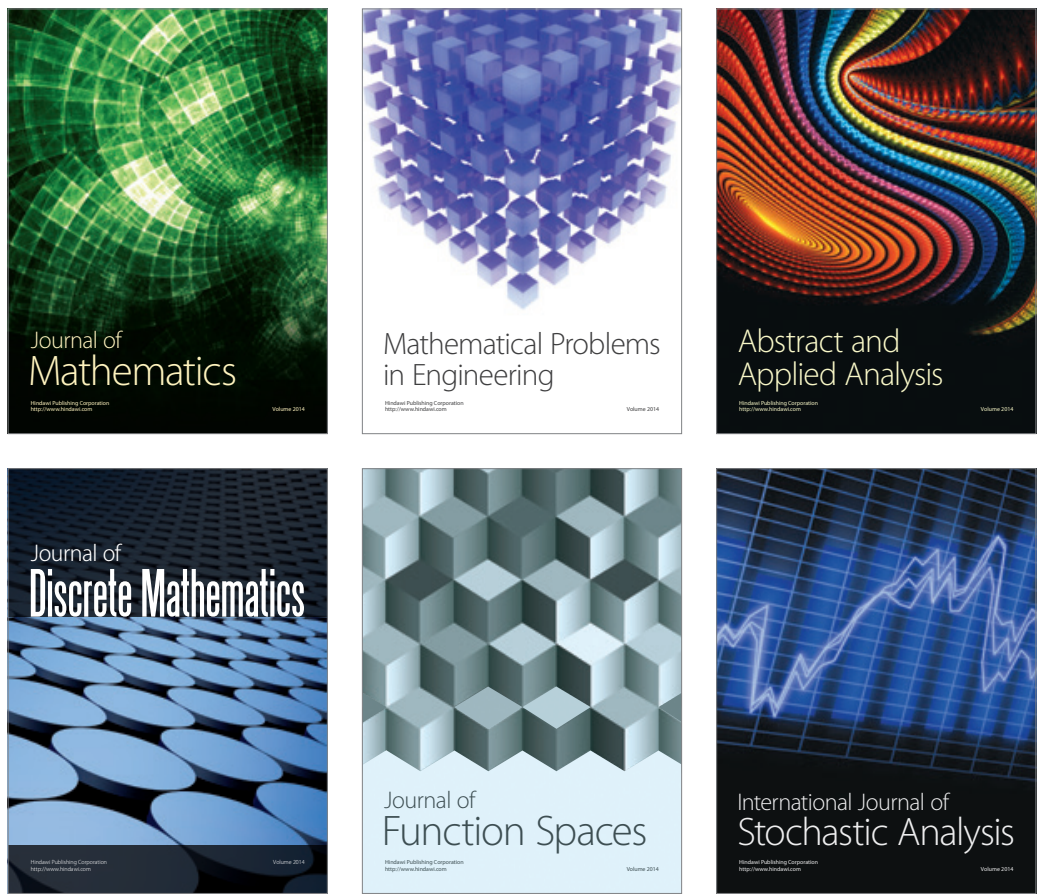

Journal of

Function Spaces

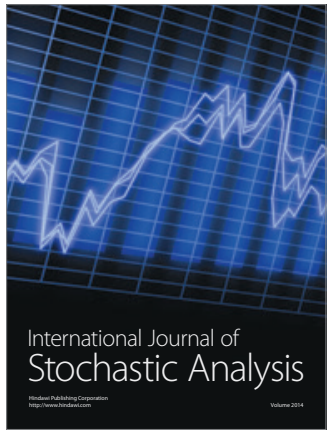

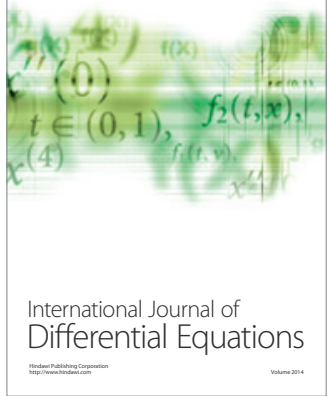
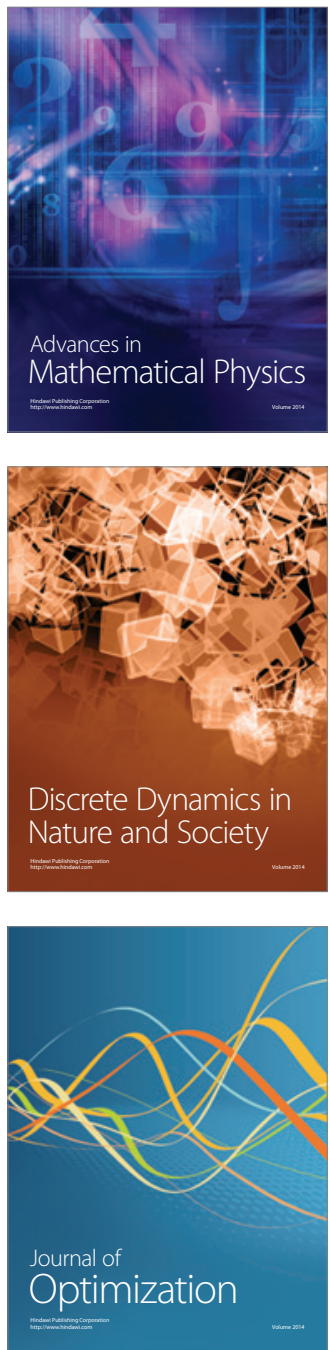